\title{
Photocatalytic degradation of organophosphate pesticides (Chlorpyrifos) using synthesized zinc oxide nanoparticle by membrane filtration reactor under UV irradiation
}

\author{
Samreen Heena Khan ${ }^{1}$, Suriyaprabha R ${ }^{1}$, Bhawana Pathak ${ }^{2}$ and M.H. Fulekar ${ }^{2 *}$ \\ ${ }^{1}$ Centre for Nanoscience, Central University of Gujarat, Gandhinagar, India \\ ${ }^{2}$ School of Environment \& Sustainable Development, Central University of Gujarat, Gandhinagar, India
}

\begin{abstract}
Nanomaterial- Zinc oxide $(\mathrm{ZnO})$ has been synthesized using Zinc Nitrate Hexahydrate as precursor by Sonochemical Method. The as-synthesized ZnO was characterized to study their optical, morphological and physical properties. Fourier Transform-Infra Red Spectroscopy analysis was done for identification of functional group, Scanning Electron Microscopy- Energy Dispersive X-Ray Analysis for Morphology and Size and elemental confirmation, Transmission Electron Microscopy for determination of particle size and shape and X-Ray Diffraction for the identification of crystal structure. The material developed was used in a membrane filtration technology for degradation of selected pesticide. Pesticides(Chlorpyrifos) taken at fixed concentration (5 ppm) in a solvent and then stimulated effluents were subjected to photocatalytic degradation and allow to pass through the membrane filtration assembly under low pressure using cellulose acetate mixed polymeric membrane discs. Samples were analyzed by UV-VIS spectroscopy for the assessment of degradation of Chlorpyrifos. Results shows that ZnO alone show low degradation efficiency as compared to membrane filtration. When combined both the approaches $(\mathrm{ZnO}+\mathrm{Membrane}$ filtration) there is steep decline in the absorption peak. Nanobased membrane filtration technology design and developed were found effective and efficient for the degradation of pesticides as compared to photocatalytic degradation carried out using $\mathrm{ZnO}$.
\end{abstract}

\section{Introduction}

Pesticide pollution emerges as the serious environmental concern. Degradation and mineralization of pesticide contaminants has becomes the key concern of scientific community. The main cause of pesticide pollution is the excessive use of pesticide, by-products and intermediated form during the degradation [1]. This persistent pollution mainly arises due to incomplete degradation by the conventional techniques, because of their high chemical stability and low biodegradability and high persistence in the atmosphere $[2,3]$.

Photocatalytic degradation by semiconducting nanoparticles is mainly considered as potential and effective technology for the mineralization of pesticide into environmental friendly compounds [4]. Nanoparticles have emerged as sustainable alternatives to conventional bulk materials, as robust, high surface area heterogeneous photo-catalysts and catalyst supports [5]. The nano-sized particles have high surface to volume ratio which increase the exposed surface area of the active component of the catalyst, enhances the contact between reactants and catalyst [6]. Surface area plays an important role in the photocatalytic activity, due to that focus hadbeen shifted towards the semiconducting nanomaterial because of their high surface to volume ratio [7]. A high surface-to-volume ratio (SVR) is required for a contaminant molecule to be adsorbed on to the surface of photocatalyst for the redox reactions (Oxidation and Reduction) to occur for the complete degradation/mineralization of the contaminant $[8,9]$. Higher the catalytic surface area, higher will be the adsorption of target molecules on the surface, leading better photocatalytic activity and complete degradation of contaminant [10]. The potential application of semiconducting materials for solar energy conversion, production and remediation is now realized by the scientific community [11]. $\mathrm{TiO}_{2}$ and $\mathrm{ZnO}$ are found to be best among all photocatalyst because of their wide band gap energy, biological and chemical inertness, ease of synthesis and applicability [12]. Among various semiconducting nanomaterials, zinc oxide $(\mathrm{ZnO})$ is a distinctive wurtzite n-type semiconductor with a wide direct band gap of $3.37 \mathrm{eV}$ and a high exciton binding energy $(60 \mathrm{meV})$ at room temperature [13]. The complete degradation of organic pollutants is not possible by conventional approaches such as anaerobic digestion, activated sludge digestion, physicochemical treatment, and air stripping as they only transfers the contaminants from one phase to another. At present, there are several methods available for pesticide removal such as advanced oxidation processes, photocatalytic degradation (heterogeneous catalysis), combined photo-Fenton (homogeneous catalysis), biological oxidation (microbial degradation), aerobic degradation, membrane filtration (by nanofiltration membranes), coagulation, solid phase extraction, ozonation, fluid extraction and adsorption [14]. Also the main method of pesticide disposal is incineration, which is very expensive and generally not available in developing nations. Thus there is need for the alternative method for complete mineralization of pesticides into

Correspondence to: M.H. Fulekar, School of Environment \& Sustainable Development, Central University of Gujarat, Gandhinagar, India, Tel: +912226528847 Fax: +91-2226526053, E-mail: mhfulekar@yahoo.com

Key words: zinc oxide, pesticides, membrane filtration, photocatalytic degradation, chlorpyrifos

Received: September 30, 2015; Accepted: October 30, 2015; Published: November 06, 2015 
the environmental friendly compounds. Out of different methods, Advance Oxidation Process (AOP) using nano based semiconductors as a photocatalyst for the degradation of pesticides is considered as most efficient, promising and environmental friendly technique [15]. Among Advance Oxidation Processes, heterogeneous photocatalysis or semiconductor mediated photocatalysis appears the most emerging destructive technology for the remediation of organic contaminants $[10,16]$. The drawback associated with the photocatalysis is the retrieval of photocatalyst after the reaction, because of this the alternative to this approach which can degrade and filtrate both at the same time [17]. The use of membrane filtration for the pesticide removal is not new but the approach combine with photocatalysis is novel for the efficient degradation. Membrane filtration is gaining importance in pesticide degradation; generally nanofiltration membrane is utilized for the same $[18,19]$. The main disadvantage of photocatalytic degradation is to retrieve the photocatalyst after degradation is over; the very quantity of catalyst is present in the treated effluent even after centrifugation [5]. The objective behind using the membrane filtration is to recover the catalyst after the degradation purpose. The study of reproducibility of the photocatalyst can be done after drying. There are very limited studies till date considering the recycling of catalyst material [20].

The present study focuses on the synthesis of $\mathrm{ZnO}$ nanoparticles by sonochemical method using Zinc Nitrate Hexahydrate $\left(\mathrm{Zn}\left(\mathrm{NO}_{3}\right)_{2}\right.$. $\left.6 \mathrm{H}_{2} \mathrm{O}\right)$ as a precursor of zinc and the characterization of $\mathrm{ZnO}$ by different characterizing tools. Sonochemical method is based on the acoustic cavitation from the continuous formation and collapse of the bubbles in a liquid [21]. This method is basically known to produce nanocrystals under the appropriate condition. Technical grade Chlorpyrifos (EC $50 \%)$ is taken as a model pesticide for the degradation studies. Mixed cellulose acetate polymeric membrane discs is used for the filtration purpose under low pressure. After photocatalytic degradation the treated effluent is allowed to pass through the membrane in a filtration assembly under low pressure. UV-Vis spectroscopy is used to study the degradation efficiency of the selected pesticide. Reaction process was optimized by taking the varying concentration of catalysts and pesticide.

\section{Structure of chlorpyrifos and mode of action}

Chlorpyrifos is basically an Organophosphate group of insecticide that inhibits the acetyl cholinesterase enzyme associated with the functioning of nervous system. It is wide spectrum insecticide thoroughly used to kill wide range of insect's pest in agriculture worldwide [22]. OP compounds are basically target the nervous system; sometime it affects the non-target group of organisms also [23]. It is highly toxic for aquatic organisms even the low concentration like parts per trillion. Symptom associated with the chlorpyrifos poisoning including nausea, vomiting, diarrhea, headache, convulsions, coma and death in severe conditions [24]. The general structural formula of chlorpyrifos is O,Odiethyl O-3,5,6-trichloro-2 pyridylphosphorothioate (Figure 1).

\section{Materials and methods}

\section{Chemicals}

Analytical grade Zinc Nitrate Hexahydrate $\left(\mathrm{Zn}\left(\mathrm{NO}_{3}\right)_{2} .6 \mathrm{H}_{2} \mathrm{O}\right)$, Sodium Hydroxide $(\mathrm{NaOH})$ (Sigma Aldrich) pellets were purchased and used without further purification. Zinc oxide nanoparticles were synthesized using sonochemical chemical method using zinc nitrate hexahydrate as precursors. Sonochemical method was selected for the synthesis of $\mathrm{ZnO}$ nanoparticle because it provides nanoparticle of

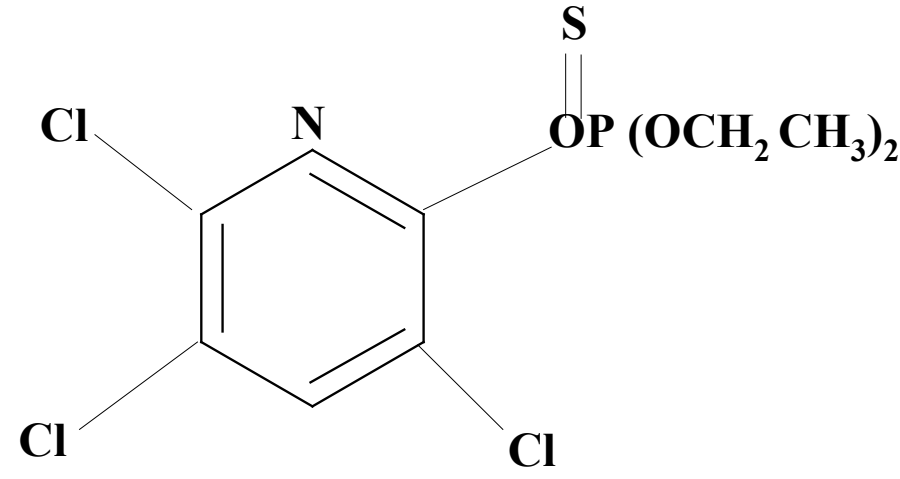

Figure 1. Structure of Chlorpyrifos.

appropriate size. Commercially available technical grade Chlorpyrifos (EC 50\%) is used for the degradation study.

\section{Experimental method}

All the reactions were carried out at room temperature under ambient conditions.

Synthesis of $\mathrm{ZnO}$ nanoparticles: In a typical reaction, $0.5 \mathrm{M}$ of Zinc Nitrate Hexahydrate $\left(\mathrm{Zn}\left(\mathrm{NO}_{3}\right)_{2} .6 \mathrm{H}_{2} \mathrm{O}\right)$ was dissolved in $100 \mathrm{ml}$ distilled water under continuous stirring. $0.5 \mathrm{M} \mathrm{NaOH}$ is prepared in different reaction vessel and stir it continuously until it gets dissolved. $\mathrm{NaOH}$ solution was added drop wise to the Zinc nitrate solution under continuous stirring until white precipitates formed. Seal the vessel and reaction is allowed to continue for 3 hours of vigorous stirring. After that the solution was kept in Ultra-sonication for 1 hour at $37^{\circ} \mathrm{C}(20-40 \mathrm{kHz})$. After sonication, the precipitates was centrifuged and washed with double distilled water several times and finally with ethanol to completely remove all the impurities. The precipitates were dried at $100^{\circ} \mathrm{C}$ for complete reduction into $\mathrm{ZnO}$ nanoparticle. Grind the powder in mortar and pestle to obtain the fine powder of $\mathrm{ZnO}$ and store it for further experiment.

\section{Customized photocatalytic reactor and filtration assembly}

All the photocatalytic experiment was conducted in a rectangular borosil glass reactor that is customized in fixed dimensions. Two UV lamp of 11W (Philips) having wavelength of $365 \mathrm{~nm}$ were used as a source of UV in the customized photo reactor fitted over the roof of the reactor. Few holes were drilled on the side of the reactor chamber to maintain the proper aeration and temperature. All the process was carried out under strict lab conditions. A filtration assembly equipped with pressure pump is utilized for the membrane filtration reaction simultaneously (Figure 2).

\section{Photocatalytic degradation studies}

In present work, photocatalytic degradation of pesticide has been measured under UV light irradiation in aqueous dispersion of synthesized $\mathrm{ZnO}$ to find the optimum reaction condition for a photocatalytic membrane filtration reactor (PMFR). The advantage of using the mixed cellulose acetate (MCA) polymeric membrane is to recover the $\mathrm{ZnO}$ photocatalyst after filtration. Low pressure ultrafiltration using hollow-fiber MCA membrane was applied for present study. The photocatalysis result indicates that the $\mathrm{ZnO}$ exhibits good degradation efficiency but when it combines with the membrane filtration the degradation efficiency increases manifolds. Dark adsorption studies were conducted with chlorpyrifos to correlates the 


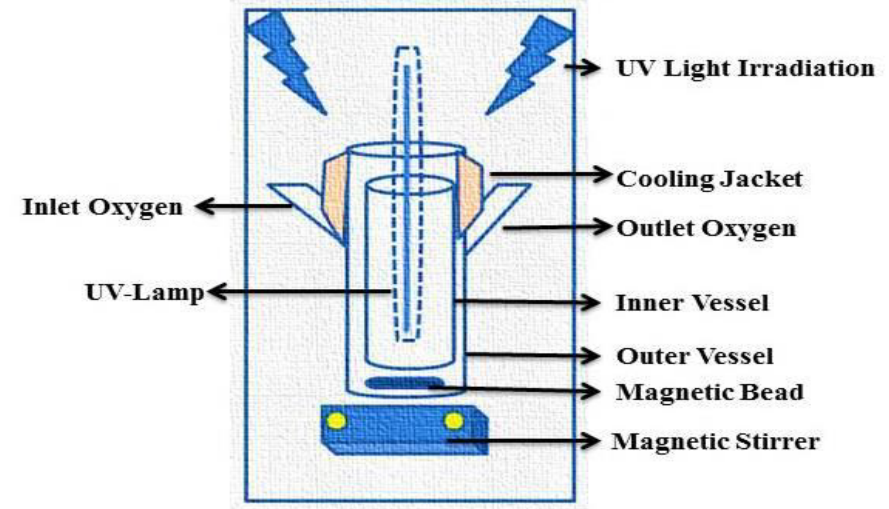

Figure 2. Schematic representation of Photocatalytic Reactor.

adsorption and degradation results (i.e. photolysis and photocatalysis). The optimized concentration of pesticide solution was prepared in distilled water. The amount of catalyst was fixed during the whole reaction whereas the concentration of the pesticide was kept varying. A volume of $250 \mathrm{ml}$ of stock solution (5 ppm) is prepared and taken in the reactor and fixed amount of catalyst was added. The suspension was first stirred under total dark condition for half an hour to reach the adsorption maximum. The stirring rate of $500 \mathrm{rpm}$ was maintained for all the experiments. Now the solution was subjected under UV irradiation with vigorous stirring in the reactor chamber by magnetic stirrer for fixed time period. An aliquot of 5-10 ml sample were taken at regular time intervals with the help of sample syringe. The sample was then pass through the $0.45 \mathrm{Mm}$ membrane filter to remove the particle and agglomerates. At last, the sample were pass through the membrane filtration assembly under low pressure after the degradation was over. The degradation efficiency of pesticide was studied using the UV-VIS spectrophotometer.

\section{Result and discussion}

\section{Characterization}

The X-Ray diffraction patterns were recorded on Bruker X-Ray Diffractometer using graphite filtered $\mathrm{CuK}$ radiation $(\lambda=1.54 \AA)$ at 40 $\mathrm{KV}$ with scanning rate of $3 / \mathrm{min}$ (from $2 \theta=20-80^{\circ}$ ). Optical absorption spectra were recorded on a UV-Vis spectrometer (Shimadzu). Size and Morphology of the particles were determined by the $200 \mathrm{KeV}$ Transmission Electron Microscope (TECNAI $200 \mathrm{Kv}$ TEM- Fei, Electron Optics). Fourier Transmission Infrared spectroscopy was performed to determine the functional group of the obtained products (Perkin Elmer- Spectrum RX-IFTIR). Elemental Detection of the particle was carried out separately using SEM-EDAX (JEOL JSM 5600,EDS Model: INCA Oxford).

Fourier Transform-Infra Red Spectroscopy (FT-IR): FT-IR studies were performed to verify the bond structure and identification of associated functional groups of as-synthesized $\mathrm{ZnO}$ nanoparticles using optimized parameters. The infrared absorption spectra of $\mathrm{ZnO}$ nanoparticles were observed in the $4000-400 \mathrm{~cm}^{-1}$ wave-number range (Figure 3). The band located at $436 \mathrm{~cm}^{-1}$ attributed to the $\mathrm{Zn}-\mathrm{O}$ stretching mode of the $\mathrm{ZnO}$ lattice [25]. The bands at 3466 $\mathrm{cm}^{-1}$ attributed to the $\mathrm{O}-\mathrm{H}$ mode of vibration. $\mathrm{C}=\mathrm{O}$ exhibiting strong asymmetric mode of vibration between 1633 and $1558 \mathrm{~cm}^{-1}$. The symmetric stretching occurs between 1508 and $1378 \mathrm{~cm}^{-1}$ because of presence of C-O. C-O-C peak is also present there.
Scanning Electron Microscopy- Energy Dispersive X-Ray Analysis (SEM-EDX): Morphological structure of as-synthesized $\mathrm{ZnO}$ was confirmed by SEM and the elemental composition of the particles was confirmed by EDAX [26]. The SEM-EDS micrograph of $\mathrm{ZnO}$ nanoparticles is shown in Figure 4. The EDAX spectra confirm the presence of $\mathrm{Zn}$ and $\mathrm{O}$ and no other impurity were detected.

X-Ray Diffraction (XRD): Figure 5 shows the XRD spectrum of

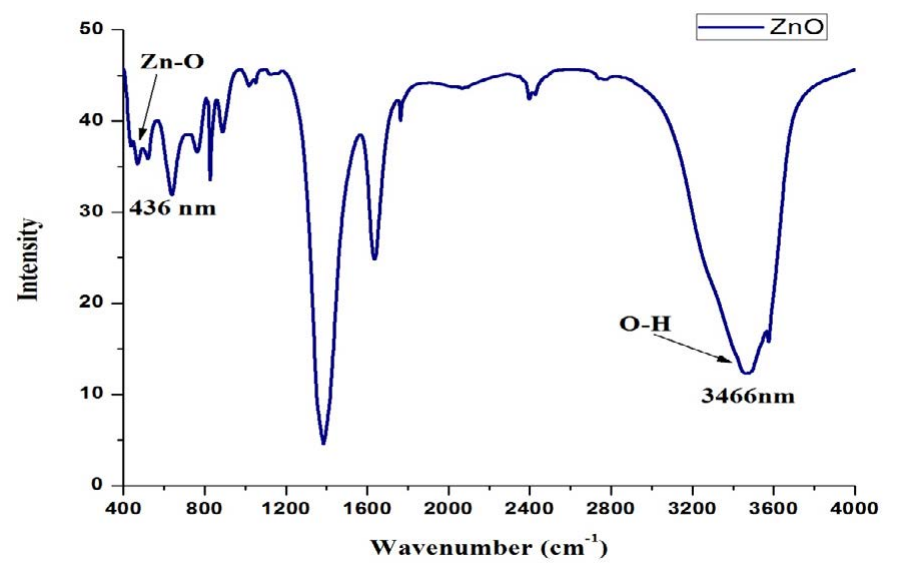

Figure 3. FT-IR spectra of $\mathrm{ZnO}$.

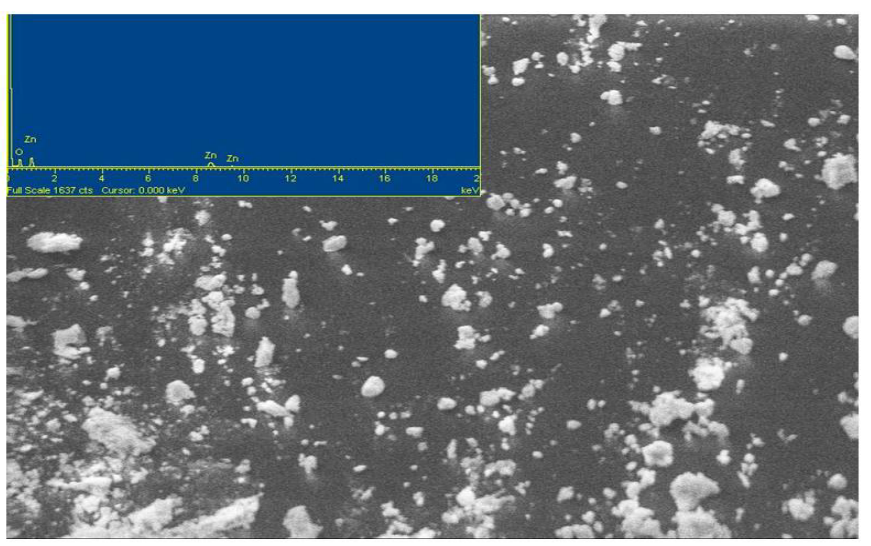

Figure 4. SEM Micrograph of as-synthesized $\mathrm{ZnO}$ (Inset- EDS spectra).

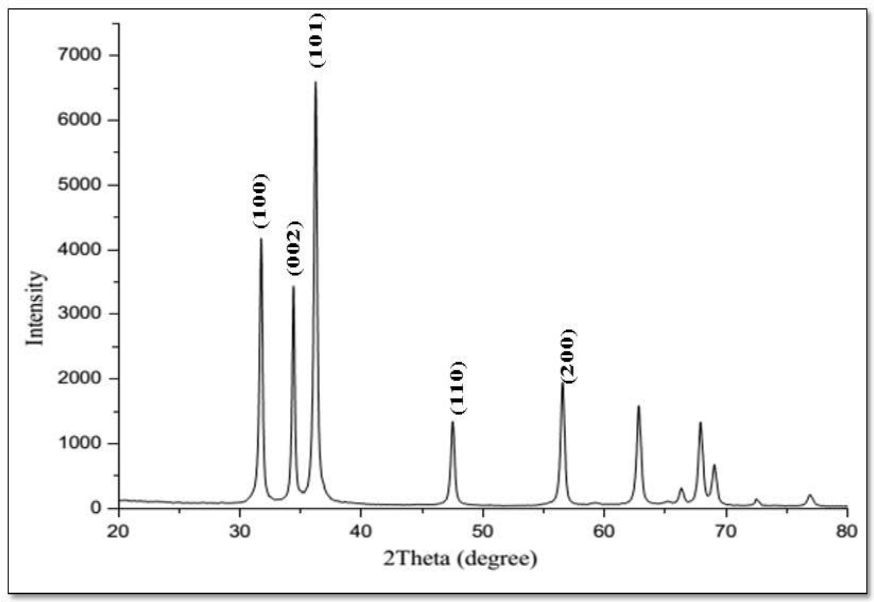

Figure 5. XRD Diffractogram of $\mathrm{ZnO}$ 

under UV irradiation

pure Zinc Oxide. The narrow and sharp diffraction peak indicates the particles have good size and crystallinity [27]. The diffraction angle of $2 \theta$ scanned in the range of $20-80^{\circ}$. The representative peaks appeared at $31.71,34.37,36.19,47.49$, and 56.51 , revealed the reflecting planes at (100), (002), (101), (110), and (200) respectively. All the diffraction peaks shows the strong resemblance with the reported Joint Committee on Powder Diffraction Standards (JCPDS) belonging to hexagonal wurtzite crystal phase of $\mathrm{ZnO}$.

Transmission Electron Microscope (TEM): To know the actual size and morphology of the particles, samples were systematically analyzed by TEM. The TEM image of $\mathrm{ZnO}$ shows the formation of spherical aggregates with dimension of $\sim 50 \mathrm{~nm}$. The actual size of the nanoparticles was found to be around $26 \mathrm{~nm}$. TEM image confirms that the morphology of nanoparticle were spheroid (Figure 6) [28,29].

Ultraviolet- Diffuse Reflectance Spectroscopy (UV-DRS): The sample shows strong excitonic peaks with the same absorbance intensity at wavelength of $380 \mathrm{~nm}$. The optical band gap of the powdered samples was characterized by using DRS [8]. The optical band gap of synthesized material was found to be $3.23 \mathrm{eV}$ which is very close to the bulk $\mathrm{ZnO}$ [30] calculated using Kubelka-Munk Transformation (Figure $7 \mathrm{a}$ and $7 \mathrm{~b}$ ).

The energy bang gap of the $\mathrm{ZnO}$ was determined using the following relationship:

$$
\begin{aligned}
& \boldsymbol{\alpha h v}=\left[\mathbf{A}\left(\mathbf{h v}-\mathbf{E}_{\mathrm{bg}}\right)\right] \\
& (\boldsymbol{\alpha h v})^{2}=\mathbf{A}\left(\mathbf{h v}-\mathbf{E}_{\mathrm{bg}}\right)
\end{aligned}
$$

Where, $h v$ is the energy of photon, $\alpha$ is the absorption coefficient, $\mathrm{E}_{\mathbf{b g}}$ is the energy band gap and $\mathrm{A}$ is the constant.

Photocatalytic-Membrane Filtration Studies: For photolysis studies, required quantity of 5 ppm chlorpyrifos (EC 50\%) was stirred
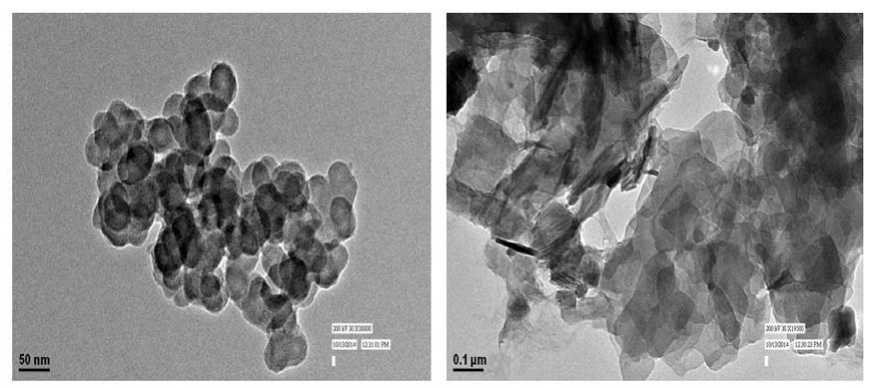

Figure 6. TEM Micrograph of $\mathrm{ZnO}$

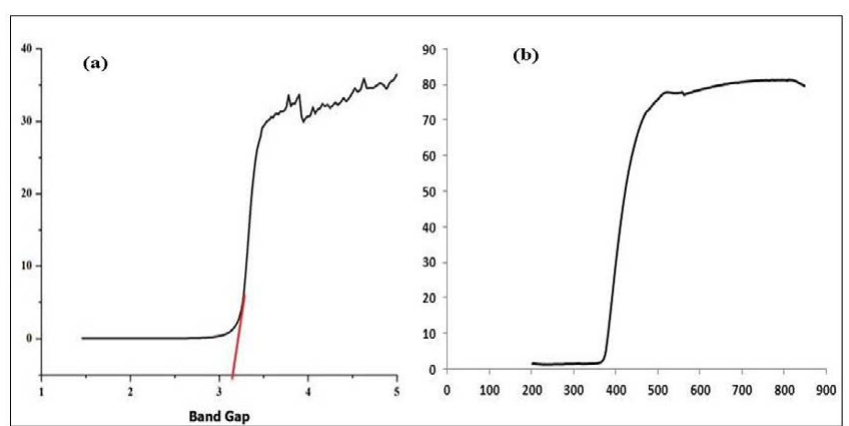

Figure 7. a) Optical bang gap of $\mathrm{ZnO}$; b) Diffuse Reflectance Spectra of as-synthesized $\mathrm{ZnO}$.
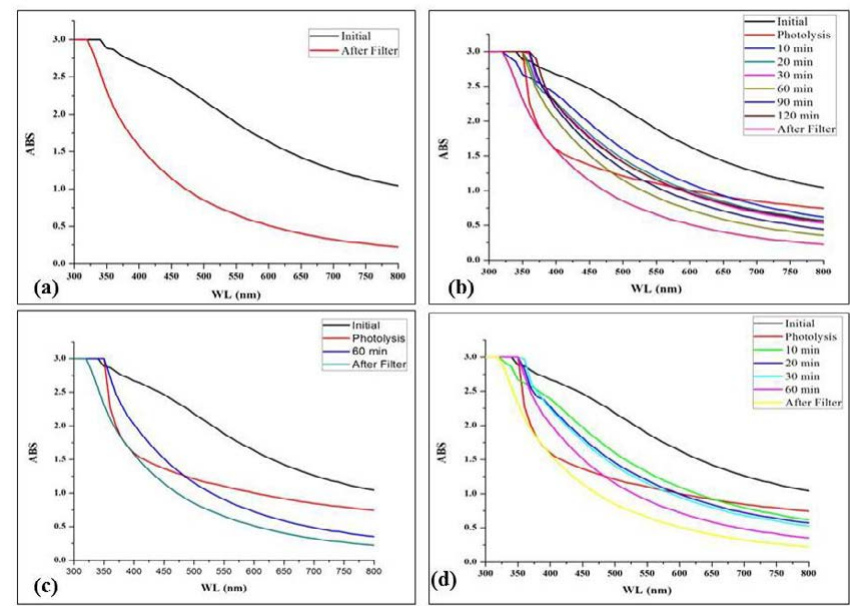

Figure 8. Plot showing the UV absorption spectra of rapid degradation of chlorpyrifos at before and after filtration $(a, b, c \& d)$.

for many hours after the addition of catalyst under dark condition. As soon as, the photocatalyst was added to the solution, pesticide starts to adsorb on the surface of the catalyst. It can be easily understood that there is a difference in the degradation efficiency after using membrane filtration as compared to $\mathrm{ZnO}$ mediated photocatalysis [31-34]. The initial absorption spectra of $5 \mathrm{ppm}$ chlorpyrifos and the final absorption spectra after filtration can be easily understood by Figure 8 (a). It is concluded that the maximum degradation is achieved in 60 minutes; the plot shows decrease in degradation efficiency after 60 minutes. The comparative plot of initial, photolysis, 60 minutes of degradation and final filtration is shown in Figure 8 (c). Thus, it was confirmed from the spectrophotometric studies which shows that the membrane filtration is a good approach when combine with the photocatalysis for the degradation of pesticides. It can also be utilized for the degradation of other contaminants as well $[35,36]$.

\section{Conclusion}

Zinc oxide nanoparticle has been successfully synthesized by sonochemical method, characterized and used as a photocatalyst for the degradation of pesticide. The results confirms the synthesis of $\mathrm{ZnO}$ under the nano range $(1-100 \mathrm{~nm})$ having spherical shape and hexagonal wurtzite crystal structure. The photocatalytic results of the as-synthesized $\mathrm{ZnO}$ indicates that the $\mathrm{ZnO}$ exhibit good photocatalytic efficiency under UV light irradiation but when combine with membrane filtration; degradation rate increases several time as compared to $\mathrm{ZnO}$ mediated photocatalysis. The results confirmed that at $5 \mathrm{ppm}$, maximum degradation achieved in 60 minutes and further degradation is achieved after membrane filtration. Present study confirmed that the Membrane filtration combined with photocatalytic degradation is efficient and time savvy technique for the degradation of pesticide compounds.

\section{Acknowledgement}

This work was supported by the University Grant CommissionMaulana Azad National Fellowship under Grant [MANF/201213/MUS/MAD/12852]. The authors are also thankful to Central Instrumentation Facility, CUG, Gandhinagar, SAIF- Punjab University, Chandigarh, SAIF- AIIMS, New Delhi, Dr. Uday Deshpande and Dr. D.M. Phase, UGC-CSR, Indore for extending analysis facility. 
Khan SH (2015) Photocatalytic degradation of organophosphate pesticides (Chlorpyrifos) using synthesized zinc oxide nanoparticle by membrane filtration reactor under UV irradiation

\section{References}

1. Cui HM, Liu H, Wang JY, Li X, Han F, Boughton RI (2004) Sonochemical synthesis of bismuth selenide nanobelts at room temperature. J Cryst Growth 271: 456-461.

2. Peng HL,Lai KJ,Kong DS,Meister S,Chen YL, et al. (2010) Aharonov-Bohm interference in topological insulator nanoribbons. Nat Mater 9: 225-229. [Crossref]

3. Tang H,Liang D,Qiu RLJ, Gao XPA (2011) Two-dimensional transport-induced linear magneto-resistance in topological insulator Bi2Se3 nanoribbons. ACS Nano 5: 75107516. [Crossref]

4. Xiu FX, He L, Wang Y,Cheng YL, Chang LT, Lang MR, et al. (2011) Manipulating surface states in topological insulator nanoribbons. Nanotech 6: 216-22. [Crossref]

5. He L, Xiu FX, Wang Y, Alexei V, Fedorov G, et al. (2011) Epitaxial growth of Bi2Se3 topological insulator thin films on Si. J Appl Phys 109: 103702

6. Tabor P, Keenan C, Urazhdin S, Lederman D (2010) Plasmon-enhanced electronphonon coupling in Dirac surface states of the thin-film topological insulator Bi2Se3. Nature Physics 6: 584-588.

7. Zhang GQ, Wang W, Lu XL, (2009) Solvothermal Synthesis of V- VI Binary and Ternary Hexagonal Platelets: The Oriented Attachment Mechanism. Growth Des 9: 145-150.

8. Checkelsky JG, Hor YS, Liu MX, Qu DX, Cava RJ, Ong NP (2009) Quantum interference in macroscopic crystals of nonmetallic Bi 2 Se 3. Phys Re Lett 103: 246601. [Crossref]

9. Alegria LD, Schroer MD, Chatterjee A, Poirier GR, Pretko M, et al. (2012) Structura and Electrical Characterization of Bi2Se3 Nanostructures Grown by Metal-Organic Chemical Vapor Deposition. Nano Lettr 12: 4711-4714. [Crossref]

10. Dang WH, Peng HL, Li HL, Wang P, Liu ZF (2010) Epitaxial heterostructures of ultrathin topological insulator nanoplate and graphene. Nano Lett 10: 2870 -2876. [Crossref]

11. Liu FY, Liu M, Liu AH, Yang C, Chen CS, et al. (2015). J Mater Sci 26: 3881-3886

12. Zhang C, Liu M, Man BY, Jiang SZ, Yang C, Chen CS, et al. (2014) Cryst Eng Comm 16: 8941 .

13. Liu M, Liu FY, Man BY, Bi D, Xu XY (2014) Multi-layered nanostructure Bi 2 Se 3 grown by chemical vapor deposition in selenium-rich atmosphere. Appl Surf Sci 317 257-261.
14. Kong DS , Cha JJ, Lai KJ, Peng HL, Analytis JG, Meister S, et al. (2011) Evaporative Thinning: A facile synthesis method for high quality ultrathin layers of 2D crystals. $\mathrm{Am}$ Chem Soc 5: 4698-4703.[Crossref]

15. Qiu Y, Wang Z, Owens AC, Kulaots I, Chen Y, et al. (2014) Antioxidant chemistry of graphene-based materials and its role in oxidation protection technology. Nanoscale 6: 11744-11755. [Crossref]

16. Denis PA, Iribarne F (2012) A First Principles Study on the Interaction between Alkyl Radicals and Graphene. Chem Eur J 18: 7568-7574. [Crossref]

17. Wang FT, Chen L, Tian CJ, Meng Y, Wang ZG, et al. (2011) Interactions between free radicals and a graphene fragment: Physical versus chemical bonding, charge transfer and deformation. J Comput Chem 32: 3264-3268. [Crossref]

18. Yang Y, Huang Y, Lv Y, Zhao P, Yang Q, et al. (2013) Mater Chem A1: 11184-11191.

19. Kim H, Miura Y, Macosko CW (2010) Graphene/polyurethane nanocomposites for improved gas barrier and electrical conductivity.Chem. Mater 22: 3441-3450.

20. Wu JR, Huang GS, Li HL, Wu, SD, Liu YF, et al. (2013) Enhanced mechanical and gas barrier properties of rubber nanocomposites with surface functionalized graphene oxide at low content. Polymer 54: 1930-1937.

21. Potts JR, Shankar O, Du L, Ruoff RS (2012) Processing-morphology-property relationships and composite theory analysis of reduced graphene oxide/natural rubber nanocomposites. Macromolecules 45: 6045-6055.

22. Tang MZ, Xing W, Wu JR, Huang GS, Xiang KW, et al. (2015) Graphene as a prominent antioxidant for diolefin elastomers. J Mater Chem A 3: 5942.

23. Chen ZS, Biscarasa J, Shukla A (2015) A high performance graphene/few-layer InSe photo-detector. Nanoscale 7: 5981. [Crossref]

24. Zhang M, Yang LQ, Yang XS , Zhao Y (2013) Materials Review 27: 7.

25. Zhang J, Peng ZP, Soni A, Zhao Y, Xiong Y, et al. (2011) Raman spectroscopy of fewquintuple layer topological insulator Bi2Se3 nanoplatelets. Nano Lett 11: 2407-2414. [Crossref]

26. Salazar-Pérez AJ, Camacho-López MA, Morales-Luckie RA, Sánchez-Mendieta V (2005) Structural evolution of Bi2O3 prepared by thermal oxidation of bismuth nanoparticles.Superficies y Vacio18: 4-8.

Copyright: (C2015 Khan SH. This is an open-access article distributed under the terms of the Creative Commons Attribution License, which permits unrestricted use, distribution, and reproduction in any medium, provided the original author and source are credited. 